\title{
A Machine Learning Algorithm for Automatic Number Plate Recognition
}

\author{
Reshu Kumari \\ Student M.Tech CSE \\ NIET Greater Noida
}

\author{
Surya Prakash Sharma \\ Assistant Professor CSE \\ NIET Greater Noida
}

\begin{abstract}
Identification of cars is a demanding function for surveillance and control systems. People can recognize automobiles through license plates which consist of alphabets and numbers. We can use the uniqueness of a combination of characters in license plates for many purposes. For example, an arrest of a suspect's vehicle, imposing parking violation fines, and entrance authentication are possible. However, it is a labor intensive job to identify all passing or parked vehicles' license plates.
\end{abstract}

This paper presents a training based approach for the recognition of vehicle number plate. The whole process has been divided into three stages i.e. capturing the image, plate localization and recognition of digits over the plate. HOG features have been used for the training purpose and Support Vector Machine is employed for the classification purpose yielding in more than $99 \%$ accuracy while recognition. The algorithm has been tested over more than 100 images.

\section{Keywords}

ANPR, SVM, HOG Features, Character Recognition.

\section{INTRODUCTION}

In recent years, license plate recognition (LPR) has become a core technology of security and traffic applications that range from traffic surveillance to parking lot access control to information management for monitoring purposes ${ }^{1}$. Simply stated, LPR helps identify vehicles and provides a reference for further vehicle tracking and activity analysis ${ }^{1}$.

Automatic license plate recognition (LPR) plays an important role in numerous applications such as unattended parking lots, security control of restricted area, traffic law enforcement, congestion pricing, and automatic toll collection ${ }^{2}$. Due to different working environments, LPR techniques vary from application to application. Most previous works have in some way restricted their working conditions, such as limiting them to indoor scenes, stationary backgrounds, fixed illumination, prescribed driveways, limited vehicle speeds, or designated ranges of the distance between camera and vehicle ${ }^{12}$. The aim of this study is to lessen many of these restrictions.

This paper is organized as following: Section 2 describes the proposed architecture and steps involved for the number plate recognition, Section 3 describes the proposed algorithm for the recognition, section 4 contains the experimental results and discussions and finally the section 5 sums up the paper with conclusion and references.

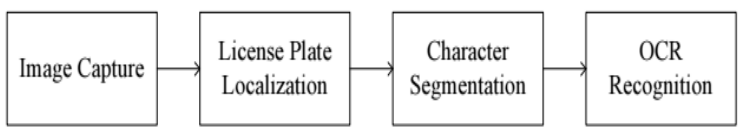

Fig.1 Architecture of the License Plate Recognition system

\section{RELATED WORK}

According to Sorin Draghici et al., an artificial neural network based artificial vision system is able to analyze the image of a car given by a camera, locate the registration plate and recognize the registration number of the car. This paper describes in detail various practical problems encountered in implementing this particular application and the methods used to solve them. The main features of the system presented are controlled stability, plasticity, behavior, controlled reliability threshold, both offline and online learning, self assessment of the output reliability and high reliability based on high level multiple feedbacks.

The system proposed by Sorin Draghici et al., has designed using a modular approach which allows easy upgrading and/or substitutions of various sub modules thus making it potentially suitable in large range of vision applications. The OCR engine was designed as an interchangeable plug-in module. This allows the user to choose an OCR engine which is suited to the particular application and to upgrade it easily in future. At present, there are several versions of OCR engine. One of them is based on fully connected feed forward artificial neural network with sigmoidal activation functions. This network can be trained with various training algorithms such as error back propagation. An alternative OCR engine is based on the constraint based decomposition (CBD) training architecture. The system has showed the following performance (on average) on real-world data successful plate location and segmentation is about $99 \%$, successful character recognition is about $98 \%$ and successful recognition of complete registration number plates of about $80 \%$.

According to Serkan Ozbay et al., and Ergun Ercelebi et al., Automatic Vehicle Identification (AVI) has many applications in traffic systems (highway electronic toll collection, red light violation enforcement, border and customs checkpoints, etc.). License Plate Recognition is an effective form of AVI systems. In their study, a smart and simple algorithm is presented for vehicle's license plate recognition system. The proposed algorithm consists of three major parts: 1. Extraction of plate region, 2. Segmentation of characters 3. Recognition of plate characters. For extracting the plate region, edge detection algorithms and smearing algorithms are used. In segmentation part, smearing algorithms, filtering and some morphological algorithms are used. Also finally statistical based template matching is used for recognition of plate characters. The performance of the proposed algorithm has been tested on real images.

Prathamesh Kulkarni et al., Ashish Khatri et al., Prateek Banga et al., Kushal Shah et al., Automatic Number Plate Recognition (ANPR) is a continuous inserted framework which consequently perceives the permit number of vehicles. In this paper, the assignment of perceiving number plate for Indian conditions is considered, where number plate gauges 
are infrequently taken after. The framework comprises of coordination of calculations like: 'Component based number plate Localization' for finding the number plate, Image Scissoring for character division and measurable element extraction for character Recognition; which is particularly intended for Indian number plates. The framework can perceive single and twofold line number plates under generally changing brightening conditions with a win rate of around $82 \%$.

\section{NUMBER PLATE RECOGNITION PROCESS}

\subsection{Image Acquisition}

Number plate recognition starts with the acquisition of images from an image source, desirably from a surveillance camera. The image acquisition technique determines the captured image quality of the number plate with which the detection algorithm have to work. Better the quality of the acquired images, higher the accuracy

\subsection{Initial Preprocessing}

A method of preprocessing is to prepare the image for better feature extraction. This can be considered as a stage to set up the vehicle picture prepared for Pattern Recognition and Image Processing. The decision of preprocessing strategy to be received on a vehicle image relies on upon the sort of use for which the image is being utilized.

\subsection{Grey Scale Conversion}

For simple examination input picture is changed over into a dark scale picture and furthermore contain less shading channels. Albeit dark scale pictures are utilized as a part of numerous NPR applications, a few examinations likewise incorporate shading pictures.

\subsection{Normalization}

Normally, the vehicle image contains text of different resolution/contrast, size and length mainly due to number plate pattern and the resolution used while acquiring the images. Because of this, the feature values extracted from such variable sized image and resolution produce inconsistent values for input images.

\subsection{Edge Enhancement}

The procured pictures require handling and upgrade before the confinement operation. The picture should be honed keeping in mind the end goal to underscore the high complexity between the picture content and the foundation.

\subsection{Segmentation}

In image processing, the segmentation of nontrivial pictures is one of the most troublesome assignments. The objective of the segmentation is to simplify or change the representation of the image or to concentrate on region of interest for the image is more important and less demanding to investigate.

\subsection{Candidate Region Generation}

The vehicle image may contain more than one areas similar to number plates and those areas are termed as candidate regions. These candidate regions may lead to the false results in segmentation of number plate. This requires the definition to gauge the normal size of the number plate.

\subsection{Feature Extraction}

Highlight extraction arrange incorporates getting the imperative information from picture remembering the true objective to diminish the limit required. Features extracted from entire image are known as the global features and those features extracted from blocks identified during segmentation or from subdivision of the number plate are as local features. They can be separated into a few classes like geometric, textural, component, segment, structural and content based.

\subsection{Classification}

The classification of vehicle number plate being handled is required for their effective revamping as it decreases number of searches, simple redesign of vehicle number plate and lessens the mistake rate at diverse phases of processing. Various computational keen architectures, for example, artificial neural networks (ANN), genetic programming (GP) and genetic algorithms (GA) were implemented for identification of number plate. In any case, these techniques need predefined parameters. The broadly utilized classifier for detection of number plate is adaptive boost and support vector machine, these methods do not need large number of parameters to get a good order execution. The SVM has been proposed in our paper.

\subsection{Enhancement of Segmented Number Plate}

Once the division of number plate picture is finished, next it is required to improve the number plate picture for encourage exercises like character division and acknowledgment.

\subsection{Noise Removal}

The segmented number plate is initially subjected for removing local noise in the number plate. Once again median filtering technique is employed on segmented number plate to make it free from noise.

\subsection{Binarization}

Binarization is a technique used to convert gray scale / color images into binary form of images. In image analysis phase it is necessary to identify the objects of interest from the input image. The system isolates the closer view and background information.

\subsection{Detection Of Oriented number Plate}

The non specific OCRs accessible are intended to peruse the content in 00 introductions and are not fit for perusing content in different introductions. The number plates portioned from caught vehicle pictures may not be in rectify introduction i.e number plate as opposed to being at 00 introduction, it might be in any of the 900,1800 and 2700 introductions due unclamped number plates toward one side and toppled vehicle. In such cases the introduction of number plate must be identified and require amendments

\subsection{Skew Detection}

The deviation of vehicle number plate to the benchmark even way is called skew. The procured vehicle number plate acquired with skew or turned by certain point is because of the camera edge amid introduction and introduction of the vehicle with individual to the camera position.

\subsection{Recognition and Interpretation}

In like manner, acknowledgment is a specialty of recognizing characters from a picture. Acknowledgment is the methodology that relegates a mark to a protest in view of the data gave by its descriptors.

Optical Character Recognition (OCR) is programmed perusing of optically recognized report content/picture materials to make an understanding of comprehensible characters to machine-significant codes. 


\section{PROPOSED SYSTEM}

\subsection{Image Capture and Resizing}

The first phase of the process is to capture the images of the moving vehicles for which number plate is to be recognized ${ }^{1}$. The captured image is then normalized to a standard size. The image is then converted to grayscale image. The next step is to localize the number plate from the captured image. Further processes are done on the localized number plate.

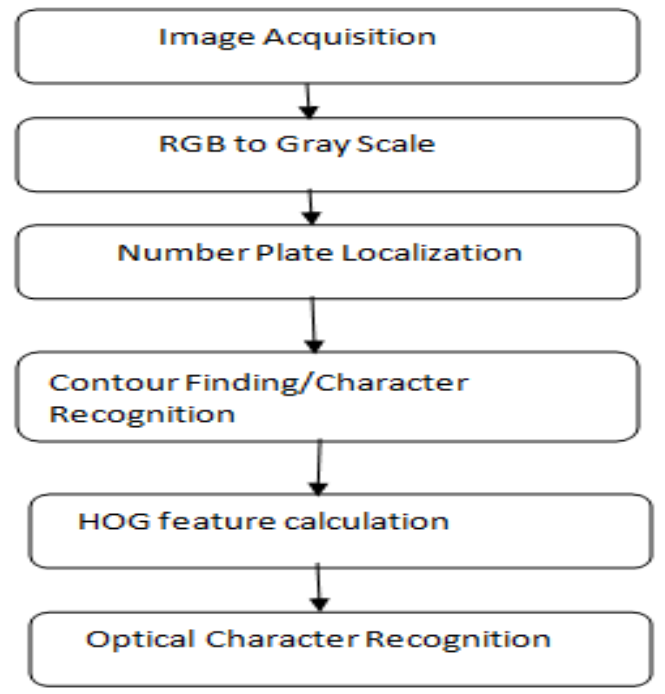

Fig. 2 Block Diagram for the Proposed Algorithm

\subsection{Training}

Image sets of 26 alphabets and 10 numbers of different sizes and shapes are created. All these binary images are then used for the training phase. During training, the Histogram of Gradient (HOG) features are calculated for all the images. Every image is also given a unique label which will be useful while classifying the alphanumeric character in the test image. The classification process is done using Support Vector Machine (SVM) classifier.

\subsection{Testing}

Proper image pre-processing techniques like converting to grayscale and adaptive histogram equalization are done to get a binary version of the test image. Connected component analysis is done on the binary image and filtered using height and width to get the alpha numeric characters in the image. Then HOG features are found for each character and it is classified using the SVM classifier to check if it belongs to the dataset we used while training. Once it passes the check, the image is cropped along the $\mathrm{Y}$ axis to get only the region where the number plate is present.

After converting the cropped image to binary, horizontal histogram is calculated. This histogram gives information about the location of the plate in the cropped image. After locating the plate in the image, again thresholding, histogram equalization is done on this image (which contains only the number plate), and subsequently connected component analysis is done and the characters are filtered using height and width. Then each valid character is then identified using Optical Character Recognition (OCR) and the final number plate characters are shown as the output

\section{EXPERIMENTAL RESULTS}

Fig. 3 shows the captured image from the moving vehicle to perform the experiment upon.

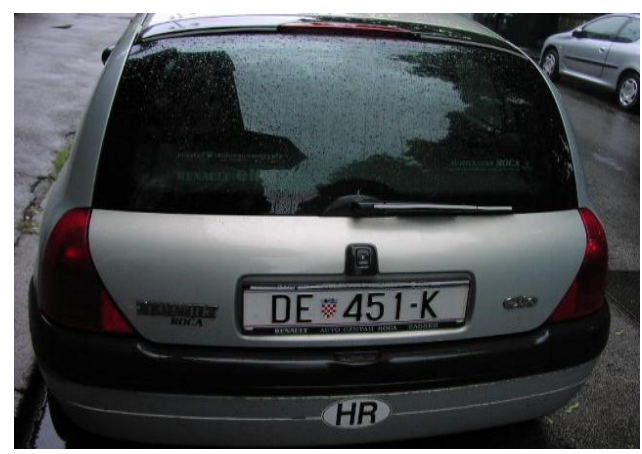

Fig 3. Image Acquisition

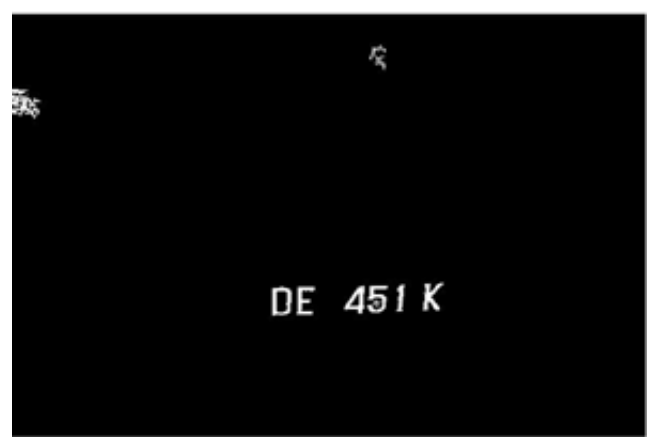

Fig4. Pre-Processed Image after Plate Localization

Fig. 4 depicts the pre processed image of the number plate after its localization i.e. extraction of number plate from the input image.

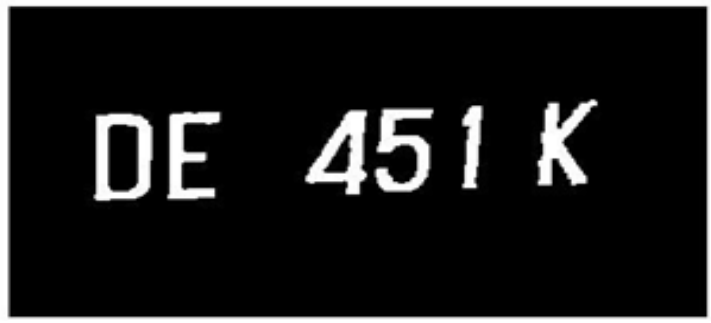

Fig5. Image filtered using Height and Width

In fig. 5 the localized number plate image has been filtered using height after applying histogram equalization on the binary image.

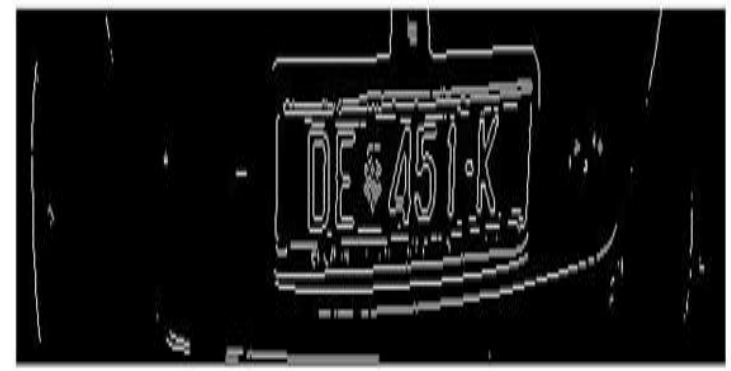

Fig.6 Edge detection 
The image is cropped along Y-axis in fig.6 for the enlarged view of the charcters.

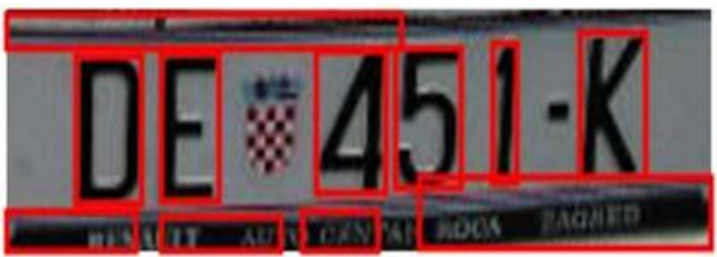

Fig 7.Cropped Number Plate

The number plate is then cropped from the whole image to get a clear image of the characters from the whole number plate.

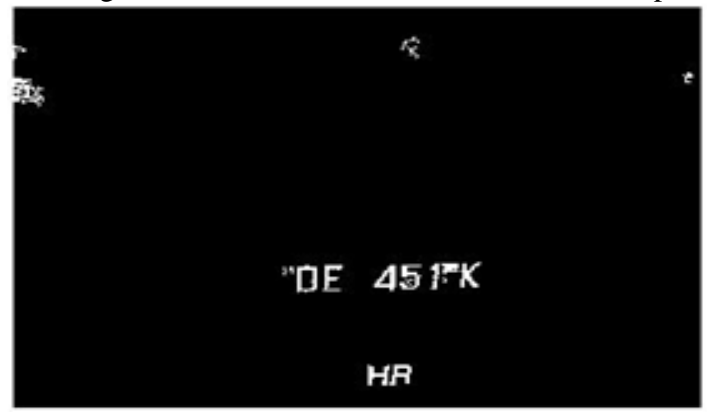

Fig.8 Processed Cropped Image

The cropped image is then processed further as shown in fig. 8 i.e. filtered using height and width.

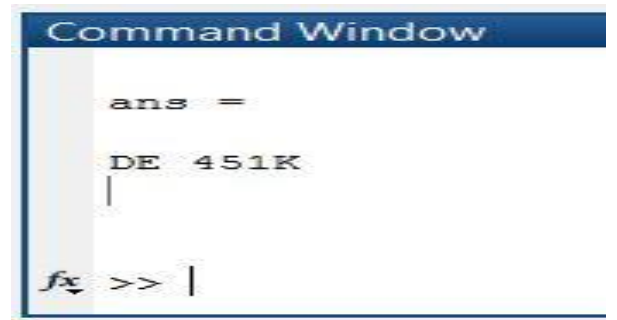

Fig.9 OCR Output of the Characters

The outputs are shown in fig. 9 after complete process.

To measure the accuracy of the algorithm, it was tested over different images and models of the cars in different lighting conditions. The plate localization accuracy was found to be almost $100 \%$, while the number plate recognition accuracy achieved was $99 \%$.

Table 1. Accuracy Comparison Results

\begin{tabular}{|l|l|l|}
\hline Methods & $\begin{array}{l}\text { Plate } \\
\text { localisation\% }\end{array}$ & $\begin{array}{l}\text { Character } \\
\text { Recognition \% }\end{array}$ \\
\hline Hitesh Rajput et. al $^{1}$ & 97.6 & 96.4 \\
\hline $\begin{array}{l}\text { M.-L. Wang and } \\
\text { colleagues }\end{array}$ & 99 & 98 \\
\hline Proposed Method & 99.8 & 99 \\
\hline
\end{tabular}

Table 1 shows the comparison between the proposed method and some recent approaches to vehicle number plate localization techniques. The results are measured in terms of accuracy.

\section{CONCLUSION}

A training based approach has been discussed in the paper for the number plate recognition. The results of the proposed algorithm signify the superiority of the proposed algorithm over some common vehicle plate recognition approaches. For the extended work this can be taken to blurred and distorted number plates and try to recognize the plates clearly in some real bad weather conditions where input images are skewed.

\section{REFERENCES}

[1] Hitesh Rajput et al.,2015, "An Automated Vehicle License Plate Recognition System," 0018- 9162 / 15/\$31.00 (C) IEEE.

[2] C.N.E. Anagnostopoulos et al.,2006, "A License PlateRecognition Algorithm for Intelligent Transportation System Applications," IEEE Trans. Intelligent Transportation Systems, vol. 7, no. 3, pp. 377-392.

[3] V. Kamat and S. Ganesan, 1997, "An Efficient Implementation of the Hough Transform for Detecting Vehicle License Plates Using DSP's, Cellular Neural Networks and Their Applications," Proc. IEEE Int'l Carnahan Conf., pp. 209-218.

[4] C.A. Rahman, W. Badawy, and A. Radmanesh,2003, "A Real-Time Vehicle's License Plate Recognition System," Proc. IEEE Conf. Advanced Video and Signal Based Surveillance (IVSS 03), pp. 163-16.

[5] L. Xiaojing, 1998, Research on Automatic Recognition of Car License Plate, Journal of Nanjing University of Aeronautics and Astronautics 30 (5), 573-576.

[6] M. N. Granieri, F. Stabile, and P. Comelli,1996 Recognition of Motor Vehicle License Plates in Rearview Images, In: Jorge L. C. Sanz (Ed.), Image Technology, Advances in Image Processing, Multimedia and Machine Vision, Springer-Verlag, Berlin, pp. 231252.

[7] M.-L. Wang et al.,2010, "A Vehicle License Plate Recognition System Based on Spatial/Frequency Domain Filtering and Neural Networks," Proc. 2nd Int' Conf. Computational Collective Intelligence: Technologies and Applications (ICCI 10), LNCS 6423, Springer, pp. 63-70.

[8] W. Wei,2001, "An Automatic Method of Location for Number-Plate Using Color Feature," Proc. IEEE Int'1 Conf. Image Processing (ICIP 01), pp. 782-785.

[9] H.W. Lim and Y.H. Tay,2010, "Detection of License Plate Characters in Natural Scene with MSER and SIFT Unigram Classifier," Proc. IEEE Conf. Sustainable Utilization Development Eng. Tech. (CSUDET 10), pp. 95-98. 